J. Dairy Sci. 97:3017-3027

http://dx.doi.org/10.3168/jds.2013-7419

(C) American Dairy Science Association ${ }^{\circledR}, 2014$.

\title{
Effect of prepubertal and postpubertal growth and age at first calving on production and reproduction traits during the first 3 lactations in Holstein dairy cattle
}

\author{
L. Krpálková, ${ }^{\star} †$ V. E. Cabrera, $\ddagger^{1}$ M. Vacek, $†$ M. Štípková, ${ }^{*}$ L. Stádník, $†$ and P. Crump§ \\ *Department of Cattle Breeding, Institute of Animal Science, Přátelství 815, 10400 Prague 10 - Uhř́něves, Czech Republic \\ †Department of Animal Husbandry, Faculty of Agrobiology, Food and Natural Resources, Czech University of Life Sciences Prague, \\ Kamýcká 129, 165 21, Prague 6 - Suchdol, Czech Republic \\ ‡Department of Dairy Science, and \\ §College of Agricultural and Life Sciences, University of Wisconsin, 1675 Observatory Dr., Madison 53716
}

\section{ABSTRACT}

The objective of this study was to evaluate the effect of body condition score (BCS), body weight (BW), average daily weight gain (ADG), and age at first calving (AFC) of Holstein heifers on production and reproduction parameters in the 3 subsequent lactations. The data set consisted of 780 Holstein heifers calved at 2 dairy farms in the Czech Republic from 2007 to 2011. Their BW and BCS were measured at monthly intervals during the rearing period (5 to 18 mo of age), and the milk production and reproduction data of the first 3 lactations were collected over an 8-yr period (2005 to 2012). The highest milk yield in the first lactation was found in the group with medium ADG (5 to 14 mo of age; 0.949 to $0.850 \mathrm{~kg}$ of $\mathrm{ADG}$ ). The highest average milk yield over lifetime performance was detected in heifers with the highest total ADG $(\geq 0.950 \mathrm{~kg} / \mathrm{d})$. The difference in milk yield between the evaluated groups of highest ADG (in total and postpubertal growth $\geq 0.950$ $\mathrm{kg} / \mathrm{d}$ and in prepubertal growth $\geq 0.970 \mathrm{~kg} / \mathrm{d}$ ) and the lowest ADG $(\leq 0.849 \mathrm{~kg} / \mathrm{d})$ was approximately 1,000 $\mathrm{kg} / 305 \mathrm{~d}$ per cow. The highest milk yield in the first lactation was found in the group with the highest AFC $\geq 751 \mathrm{~d}$, for which fat and protein content in the milk was not reduced. Postpubertal growth (11 to 14 mo of age) had the greatest effect on AFC. The group with lowest AFC $\leq 699 \mathrm{~d}$ showed a negative effect on milk yield but only in the first $100 \mathrm{~d}$ of the first parity. The highest ADG was detrimental to reproduction parameters in the first lactation. The highest BW at 14 mo $(\geq 420 \mathrm{~kg})$ led to lower AFC. Groups according to BCS at 14 mo showed no differences in AFC or milk yield in the first lactation or lifetime average production per lactation. We concluded that low AFC $\leq 699 \mathrm{~d}$ did not show a negative effect on subsequent production and

Received August 26, 2013

Accepted January 10, 2014.

${ }^{1}$ Corresponding author: vcabrera@wisc.edu reproduction parameters. Therefore, a shorter rearing period is recommended for dairy herds with suitable management.

Key words: average daily weight gain, body condition score, calf performance, heifer performance

\section{INTRODUCTION}

Age at first calving (AFC) is an important factor in dairy herd replacements and can be manipulated by altering growth rates (Ettema and Santos, 2004). The biological interrelationships between growth rate and subsequent reproduction and the ability to produce milk appear to be of great importance for a final economic evaluation of a reduced rearing period (Mourits et al., 1999a). Feeding intensity during rearing can be divided into periods before and after sexual maturity (Abeni et al., 2000; Shamay et al., 2005; Daniels, 2010). One strategy for reducing the costs of milk production could be to shorten the rearing period. Thus, heifers could calve when they are no more than 24 mo old and have adequate body size (Mourits et al., 1999b; Gabler and Heinrichs, 2003; Shamay et al., 2005; Stevenson et al., 2008). This goal requires an ADG of between 0.7 and $0.8 \mathrm{~kg} / \mathrm{d}$ in a large-size breed (Abeni et al., 2000). According to Shamay et al. (2005), a rapid growth rate from 3 to 12 mo of age led to a decrease in milk production, as a result of an increase in the mammary adipose tissue and its parenchymal content. Increased feeding intensity (ADG higher than $0.7 \mathrm{~kg} / \mathrm{d}$ ) before sexual maturity causes changes in the secretion of hormones in the lactogenic complex, resulting in a reduced number of secretory cells in the mammary gland (Mourits et al., 1999a; Daniels, 2010), and the most critical period is during the allometric phase (Shamay et al., 2005). Moderate feed restriction during the critical period is recommended (Ford and Park, 2001). However, when a delay was induced in the growth rate of calves, full recovery of skeletal size or BW was not achieved by 
compensatory feeding (Shamay et al. 2005). Mourits et al. (2000) found that ADG of $0.9 \mathrm{~kg} / \mathrm{d}$ in the prepubertal period and a maximum ADG of $1.1 \mathrm{~kg} / \mathrm{d}$ in the postpubertal period enabled achieving AFC of 20.5 mo. Abeni et al. (2000) found that early calving, following a moderate $(0.7 \mathrm{~kg} / \mathrm{d})$ and also an accelerated $(0.9 \mathrm{~kg} / \mathrm{d})$ ADG, negatively influenced milk production and milk fat percentage. Most reports agree that an ADG of approximately $0.7 \mathrm{~kg} / \mathrm{d}$ for Holsteins during the entire rearing period of dairy heifers is optimal for subsequent maximum milk yields per lactation, with no negative effects up to approximately $0.9 \mathrm{~kg} / \mathrm{d}$ (Zanton and Heinrichs, 2005). Nonetheless, Lucy (2001) reported negative effects of high ADG on reproductive performance.

Systematic evaluation of the growth and development of heifers is necessary for ensuring ideal management. However, BW does not reflect the level of nutrition or the capacity of the heifer's body (Le Cozler et al., 2008). To find possible deviations from optimal growth, it is necessary to evaluate the development of the skeleton (height) and fat tissue in the body (Shamay et al., 2005). Body condition score, based on a 5-unit scale, is a common method for dairy cow evaluation of fat deposition patterns that can also be used with successful results for heifers (Macdonald et al., 2005). Kadokawa and Martin (2006) reviewed the important role of adipose mass on reproduction and concluded that adipose mass increases as an organism grows. Shamay et al. (2005) determined that an average increase of 0.77 BCS units during the $60 \mathrm{~d}$ following the onset of puberty was an integral part of the puberty process. The objective of this study was to determine the effect of BCS, BW, ADG, and AFC in Holstein heifers fed similar diets on milk yield and reproduction traits in the subsequent 3 lactations.

\section{MATERIALS AND METHODS}

\section{Data}

The data set observed in this study consisted of 780 Holstein heifers that calved at 2 dairy farms in the Czech Republic during 2007 to 2011. The animals were, in general, kept under the same conditions. All the calves were housed in individual hutches equipped with buckets for water and a starter mixture. The calves had free access to water and starter mix and, subsequently, all heifers were fed the same maintenance diet and housed in a free-stall barn after weaning (approximately 3 mo of age). The diet for the heifers consisted of a TMR (a mixture of forage and grain) fed once daily. Two types of TMR were used at each farm. The diets and their nutritional contents are described in Table 1. The first type of TMR was fed up to 12 mo of age and the second type of TMR from 12 mo of age to the end of the rearing period.

All evaluated heifers were fed the same consistent feeding ration, regardless of season of calving. A liveweight of approximate $400 \mathrm{~kg}$ was crucial for the first insemination of heifers, and the approximate conception period was 14 mo of age. The height of animals was also considered as a criterion for first insemination to achieve the optimal time for breeding. Heifers were inseminated if the height of the withers reached $130 \mathrm{~cm}$, regardless of their BW. The optimal time for breeding (AI) was determined using farm records along with heat detection with a pedometer. Rapidly growing animals with good reproduction abilities achieved a lower AFC. Farm records of BCS, BW, and respective reproduction and production traits during the first 3 lactations measured within the milk recording system (ICAR, 2012) were used for evaluation. Individual milk production records during the first 3 lactations that lasted at least $250 \mathrm{~d}$ were included in the analyses. Lactations were standardized to $305 \mathrm{~d}$. Some of the heifers studied did not finish all the evaluated lactations and some were culled, but their data were used until they left the herd. Lifetime production $(\mathbf{L P})$ was then calculated as the lifetime average 305-d production per lactation (milk yield and percentage of protein and fat in milk) in addition to the production of the first 3 lactations and the milk production for the first $100 \mathrm{~d}$ of the first lactation. Body condition score was ranked on a standard 5-point scale (Edmonson et al., 1989) and BW were measured at monthly intervals during the period of 5 to 18 mo of age. Farm-trained personnel carried out measurements. Average daily weight gain was calculated using the difference in BW between 2 consecutive months during the period analyzed. Animals did not have the same age at the start of data collection and, therefore, the data set was unbalanced (Table 2). The basic characteristics of the variables in the data sets, including the number of animals with data in each lactation, are listed in Tables 2 and 3.

\section{Statistical Analysis}

The ADG of the heifers was divided into 3 evaluated periods (Table 2): prepubertal growth (5 to 10 mo of age), postpubertal growth (period after sexual maturity to insemination: 11 to 14 mo of age), and total period of growth up to the insemination period of the heifers (5 to $14 \mathrm{mo}$ of age). The explanatory variables BCS at $14 \mathrm{mo}$ of age, BW at 14 mo of age, ADG, and AFC (Table 2) were divided into groups and the classification was uniform for all the analyses (Tables 4 and 5).

Distribution of AFC (Figure 1) showed that the most common AFC ranged between 23 and 24 mo of age and, 
Table 1. Feed ingredients and nutritional contents of diets provided to heifers

\begin{tabular}{|c|c|c|}
\hline Item & $\begin{array}{l}\text { TMR from weaning } \\
\text { to } 12 \text { mo of age }\end{array}$ & $\begin{array}{l}\text { TMR from } 12 \text { mo of age to } \\
\text { the end of the rearing period }\end{array}$ \\
\hline \multicolumn{3}{|l|}{ Feed ingredient } \\
\hline Corn silage, $\mathrm{kg}$ & 9 & 9 \\
\hline Alfalfa haylage, $\mathrm{kg}$ & 7 & 3 \\
\hline Alfalfa hay, kg & 1 & 1 \\
\hline Grass hay, kg & 0.75 & - \\
\hline Barley straw, kg & 2 & - \\
\hline Blattin M92 ADE, ${ }^{1} \mathrm{~kg}$ & 0.1 & - \\
\hline COT Lany, ${ }^{2} \mathrm{~kg}$ & - & 1.7 \\
\hline \multicolumn{3}{|l|}{ Nutrient content } \\
\hline $\mathrm{ME}: \mathrm{DP},{ }^{3} \mathrm{Mcal} / \mathrm{g}$ & $36-66: 1$ & 40-45:1 \\
\hline NFD, g & 2,180 & 2,437 \\
\hline $\mathrm{ADF}, \mathrm{g}$ & 2,180 & 2,180 \\
\hline $\mathrm{CP}, \mathrm{g}$ & 1,088 & 951 \\
\hline $\mathrm{P}, \mathrm{g}$ & 23.05 & 22.66 \\
\hline $\mathrm{K}, \mathrm{g}$ & 165.30 & 101.18 \\
\hline $\mathrm{K}: \mathrm{Na}$ & 12.02:1 & 18.06:1 \\
\hline Ca:P & $3.60: 1$ & $2.25: 1$ \\
\hline
\end{tabular}

${ }^{1}$ Blattin Polska Sp. z o.o. (Izbicko, Poland). Nutritional additives per kilogram: 500,000 IU of vitamin A (E 672 ), 80,000 IU of vitamin $\mathrm{D}_{3}$ (E 671), 3,025 $\mathrm{mg}$ of vitamin E, $40 \mathrm{mg}$ of selenium [combination of $20 \mathrm{mg}$ of rumen-protected selenium $+20 \mathrm{mg}$ of fast-available selenium (sodium selenite, E 8)], $20 \mathrm{mg}$ of vitamin B1, 30 $\mathrm{mg}$ of vitamin $\mathrm{B}_{2}, 25 \mathrm{mg}$ of vitamin $\mathrm{B}_{6}, 250 \mu \mathrm{g}$ of vitamin $\mathrm{B}_{12}, 156 \mathrm{mg}$ of niacin, $67 \mathrm{mg}$ of pantothenic acid, $4 \mathrm{mg}$ of folic acid, $9,000 \mathrm{mg}$ of $\mathrm{Zn}, 3,035 \mathrm{mg}$ of $\mathrm{Mn}, 1,500 \mathrm{mg}$ of $\mathrm{Cu}, 20 \mathrm{mg}$ of Co, and $100 \mathrm{mg}$ of I. Analysis: $21.1 \%$ calcium, $3.1 \%$ phosphorus, $8.2 \%$ sodium, and $4.1 \%$ magnesium.

${ }^{2} 31 \%$ wheat, $30 \%$ barley, and $12 \%$ rapeseed meal (Czech University Life Science Farm Estate Lany, Czech Republic)

${ }^{3} \mathrm{DP}=$ digestible protein.

therefore, the age at first conception ranged between 14 to 15 mo of age. In accordance with this, BCS and BW were evaluated at $14 \mathrm{mo}$ of age (Tables 2 and 5 ), the time of the first insemination.

The MIXED procedure of SAS (SAS Institute, 2008) under the models described in Equations 1 and 2, corrected for other significant effects, was applied to determine the effect of the heifer rearing period on reproduction and production traits during the subsequent 3 lactations. The Tukey method was used for means comparison (Verbeke and Molenberghs, 2000). Models used only fixed effects and individuals were considered independent observations:

$$
\begin{gathered}
\mathrm{y}_{\mathrm{ijklmn}}=\mu+\mathrm{A}_{\mathrm{i}}+\mathrm{S}_{\mathrm{j}}+\mathrm{H}_{\mathrm{k}}+\mathrm{B}_{\mathrm{l}}+\mathrm{BV}_{\mathrm{m}} \\
+\mathbf{b}\left(\mathrm{Age}_{\mathrm{ijklm}}-\mathrm{Age}_{00000}\right)+\mathrm{e}_{\mathrm{ijklmn}},
\end{gathered}
$$

where $y_{i j k l m n}=$ the value of the dependent variable (listed in Table 3), $\mu=$ the overall mean, $A_{i}=$ effect of the ith year of calving ( $\mathrm{i}=2007,2008,2009,2010$, or 2011), $S_{j}=$ the effect of the jth season of calving $(j=$ spring, summer, autumn, or winter), $\mathrm{H}_{\mathrm{k}}=$ the effect of the kth herd, $\mathrm{B}_{1}=$ the explanatory variables (effect of the lth category of BCS, BW, or ADG, listed in Table $2), \mathrm{BV}_{\mathrm{m}}=$ the effect of the mth estimated sire breeding value for milk (kg; $\mathrm{m}=\geq 750,749-300$, or $\leq 299)$, b = the vector of regression coefficients of AFC used for $\mathrm{B}_{1}$ and only for analysis of production and reproduction traits in the first 3 lactations, Age $\mathrm{ijklm}_{\mathrm{jk}}=$ the $\mathrm{AFC}$ in days, $\mathrm{Age}_{00000}=$ the overall mean for the $\mathrm{AFC}$, and $\mathrm{e}_{\mathrm{ijk} k \mathrm{mn}}=$ the random error

The simplified Equation 2 was used for determination of the effect on selected factors on a dependent observed variable during the rearing period:

Table 2. Evaluated parameters in the rearing period of heifers (explanatory variables)

\begin{tabular}{lcrrrc}
\hline Item & $\mathrm{n}$ & \multicolumn{1}{c}{ Mean } & \multicolumn{1}{c}{$\mathrm{SD}$} & Minimum & Maximum \\
\hline BCS at 14 mo of age (5-point scale) & 530 & 3.37 & 0.33 & 2.00 & 4.50 \\
BW at 14 mo of age, $\mathrm{kg}$ & 780 & 412.49 & 37.50 & 250 & 530 \\
ADG from 5 to 10 mo of age, $\mathrm{kg} / \mathrm{d}$ & 392 & 0.91 & 0.11 & 0.54 & 1.21 \\
ADG from 11 to 14 mo of age, $\mathrm{kg} / \mathrm{d}$ & 528 & 0.91 & 0.08 & 0.62 & 1.17 \\
ADG from 5 to 14 mo of age, $\mathrm{kg} / \mathrm{d}$ & 370 & 0.91 & 0.09 & 0.58 & 1.19 \\
AFC, ${ }^{1} \mathrm{~d}$ & 780 & 727 & 58 & 581 & 1,051 \\
\hline
\end{tabular}

${ }^{1}$ Age at first calving. 
Table 3. Evaluated parameters in heifers and 3 subsequent lactations (dependent variables)

\begin{tabular}{lccccc}
\hline Item & $\mathrm{n}$ & Mean & $\mathrm{SD}$ & Minimum & Maximum \\
\hline Services per conception (heifers) & 780 & 1.95 & 1.34 & 1.00 & 9.00 \\
First lactation & & & & & \\
Milk (first 100 d), kg & 780 & 2,916 & 507 & 852 & 4,595 \\
Protein (first 100 d), \% & 780 & 3.91 & 0.53 & 2.42 & 4.46 \\
Fat, \% (first 100 d) & 780 & 3.09 & 0.19 & 2.55 & 3.74 \\
Milk (305-d lactation), kg & 780 & 8,511 & 1,296 & 3,624 & 13,475 \\
Protein, \% & 780 & 3.25 & 0.17 & 2.72 & 3.78 \\
Fat, \% & 780 & 3.85 & 0.44 & 2.67 & 5.28 \\
Services per conception & 428 & 2.35 & 1.70 & 1.00 & 10.00 \\
Days open, d & 702 & 149 & 85.24 & 39 & 525 \\
Calving interval, d & 500 & 415 & 83.28 & 267 & 801 \\
Second lactation & & & & & \\
Milk (305-d lactation), kg & 443 & 10,116 & 1,779 & 5,041 & 16,877 \\
Protein, \% & 443 & 3.24 & 0.20 & 2.77 & 4.06 \\
Fat, \% & 443 & 3.65 & 0.43 & 2.41 & 5.07 \\
Days open, d & 367 & 157 & 82.42 & 42 & 482 \\
Calving interval, d & 232 & 415 & 77.49 & 291 & 803 \\
Third lactation & & & & & 16,749 \\
Milk (305-d lactation), kg & 194 & 10,443 & 1,684 & 5,916 & 3.77 \\
Protein, \% & 194 & 3.17 & 0.17 & 2.78 & 4.60 \\
Fat, \% & 194 & 3.60 & 0.41 & 2.45 & 12,958 \\
LP & & & & 3.78 \\
Milk (305-d lactation), kg & 194 & 10,032 & 1,221 & 6,481 & 2.82 \\
Protein, \% & 194 & 3.20 & 0.16 & 2.75 & 4.86 \\
Fat, \% & 194 & 3.65 & 0.39 & &
\end{tabular}

${ }^{1}$ Lifetime average production per lactation.

$$
\mathrm{y}_{\mathrm{ijklm}}=\mu+\mathrm{C}_{\mathrm{i}}+\mathrm{D}_{\mathrm{j}}+\mathrm{H}_{\mathrm{k}}+\mathrm{B}_{\mathrm{l}}+\mathrm{e}_{\mathrm{ijklm}}
$$

where $\mathrm{y}_{\mathrm{ijklm}}=$ the value of the dependent variable [i.e., BCS at 14 mo of age, BW at 14 mo of age, insemination index per heifer (number of inseminations per pregnant heifer), and $\mathrm{AFC}], \mu=$ the overall mean, $\mathrm{C}_{\mathrm{i}}=$ the ith year of birth ( $\mathrm{i}=2005,2006,2007,2008$, or 2009), $\mathrm{D}_{\mathrm{j}}=$ the effect of the jth season of birth ( $\mathrm{j}=$ spring, summer, autumn, or winter), and $\mathrm{e}_{\mathrm{ijk} k \mathrm{~m}}=$ the random error. The differences between the variables estimated were tested at the level of significance of $P<0.05$.

\section{RESULTS}

\section{ADG and Subsequent Performance}

A significant difference $(P<0.05)$ between the highest and the lowest ADG in evaluated periods was found in relation to the AFC. The lowest AFC was achieved with the highest overall ADG $(\geq 0.950 \mathrm{~kg} / \mathrm{d})$ and prepubertal growth $(\geq 0.970)$. The AFC declined by 17,38 , and $22 \mathrm{~d}$ when the highest ADG occurred in the prepubertal, postpubertal, and total growth period, respectively. Milk yield in the first, second, and third lactations and the LP of all ADG groups $\leq 0.849 \mathrm{~kg} / \mathrm{d}$ were significantly lower $(P<0.05)$ than in other groups (Table 4). The highest milk yield in the first lactation was found in the medium group of ADG (0.969 to 0.850 $\mathrm{kg} / \mathrm{d}$ in the prepubertal growth period; 0.949 to 0.850 $\mathrm{kg} / \mathrm{d}$ in the total growth period), except during the postpubertal growth period. The milk yield reached $9,117 \mathrm{~kg} / 305 \mathrm{~d}$ per cow during prepubertal growth in accordance with the total growth intensity measured from 5 to 14 mo of age $9,289 \mathrm{~kg} / 305 \mathrm{~d}$ per cow (Table 4 ). The same trend was observed in milk yield in the first $100 \mathrm{~d}$ in the first lactation. The regression and correlation coefficients for milk yield in the first lactation versus total ADG were $1,835 \mathrm{~kg}(P<0.05)$ and $0.09(P$ $<0.05)$, respectively. The correlation between evaluated variables was low. The highest milk yield in the LP (Table 4) occurred in the group with the highest ADG $(\geq 0.950 \mathrm{~kg} / \mathrm{d}$; in prepubertal growth: $\geq 0.970 \mathrm{~kg} / \mathrm{d})$, except for during the postpubertal growth period. The differences between the highest and lowest ADG in milk yield ranged from 837 to $945 \mathrm{~kg} / 305 \mathrm{~d}$ per cow $(P$ $<0.05$ ). The regression and correlation coefficients for the milk yield of LP versus total ADG were $3,865 \mathrm{~kg}$ $(P<0.01)$ and $0.27(P<0.01)$, respectively. The level of ADG affected the content of milk components: the higher the ADG, the higher the percentage of fat and protein content. Similar differences $(P<0.05)$ in milk yield were determined even in the second and third parity (Table 4). Groups with the highest ADG showed the highest milk yield in the second and third parity, except for the postpubertal growth group in the third parity. Days open in the first and the second parity differed $(P<0.05)$ only in relation to the intensity of postpubertal growth. Groups with more days open were 
Table 4. Effects of heifer ADG on production and reproduction outcomes

$\mathrm{ADG}, \mathrm{kg} / \mathrm{d}$

\begin{tabular}{|c|c|c|c|c|c|c|c|c|c|}
\hline \multirow[b]{2}{*}{ Item } & \multicolumn{9}{|c|}{$\mathrm{ADG}, \mathrm{kg} / \mathrm{d}$} \\
\hline & $\begin{array}{c}5-10 \text { mo of age } \\
(\geq 0.970)\end{array}$ & $\begin{array}{c}5-10 \text { mo of age } \\
(0.969-0.850)\end{array}$ & $\begin{array}{c}5-10 \text { mo of age } \\
(\leq 0.849)\end{array}$ & $\begin{array}{l}11-14 \text { mo of age } \\
(\geq 0.950)\end{array}$ & $\begin{array}{c}11-14 \text { mo of age } \\
(0.949-0.850)\end{array}$ & $\begin{array}{l}11-14 \text { mo of age } \\
(\leq 0.849)\end{array}$ & $\begin{array}{c}5-14 \text { mo of age } \\
(\geq 0.950)\end{array}$ & $\begin{array}{c}5-14 \text { mo of age } \\
(0.949-0.850)\end{array}$ & $\begin{array}{c}5-14 \text { mo of age } \\
\quad(\leq 0.849)\end{array}$ \\
\hline $\mathrm{n}$ & 122 & 167 & 103 & 162 & 239 & 127 & 119 & 156 & 95 \\
\hline $\begin{array}{l}\text { BCS at } 14 \mathrm{mo}, 5 \text {-point } \\
\text { scale }\end{array}$ & $3.43 \pm 0.03^{\mathrm{a}}$ & $3.26 \pm 0.03^{\mathrm{b}}$ & $3.26 \pm 0.04^{\mathrm{b}}$ & $3.50 \pm 0.03^{\mathrm{a}}$ & $3.39 \pm 0.03^{\mathrm{b}}$ & $3.26 \pm 0.04^{\mathrm{c}}$ & $3.44 \pm 0.04^{\mathrm{a}}$ & $3.29 \pm 0.03^{\mathrm{b}}$ & $3.19 \pm 0.04^{c}$ \\
\hline BW at $14 \mathrm{mo}, \mathrm{kg}$ & $428.57 \pm 2.56^{\mathrm{a}}$ & $398.41 \pm 2.20^{\mathrm{b}}$ & $371.77 \pm 2.93^{\mathrm{c}}$ & $450.47 \pm 1.87^{\mathrm{a}}$ & $413.68 \pm 1.60^{\mathrm{b}}$ & $373.63 \pm 2.19^{\mathrm{c}}$ & $432.61 \pm 2.27^{\mathrm{a}}$ & $398.21 \pm 1.98^{\mathrm{b}}$ & $365.38 \pm 2.60^{c}$ \\
\hline $\begin{array}{l}\text { Services per conception } \\
\text { (heifers) }\end{array}$ & $1.45 \pm 0.38$ & $1.26 \pm 0.34$ & $1.40 \pm 0.35$ & $1.90 \pm 0.15$ & $1.77 \pm 0.13$ & $1.91 \pm 1.18$ & $2.19 \pm 0.18$ & $2.12 \pm 0.14$ & $2.28 \pm 0.20$ \\
\hline $\begin{array}{l}\mathrm{AFC},{ }^{1} \mathrm{~d} \\
\text { First lactation }\end{array}$ & $707.40 \pm 10.32^{\mathrm{b}}$ & $713.24 \pm 9.65^{\mathrm{b}}$ & $724.11 \pm 9.61^{\mathrm{a}}$ & $715.79 \pm 4.04^{\mathrm{b}}$ & $721.55 \pm 3.47^{\mathrm{b}}$ & $753.02 \pm 4.73^{\mathrm{a}}$ & $712.64 \pm 4.22^{\mathrm{b}}$ & $718.45 \pm 3.67^{\mathrm{b}}$ & $734.06 \pm 4.82^{\mathrm{a}}$ \\
\hline Milk (first $100 \mathrm{~d}$ ), kg & $3,013 \pm 126.16^{\mathrm{ab}}$ & $3,069 \pm 115.28^{\mathrm{a}}$ & $2,902 \pm 116.50^{\mathrm{b}}$ & $2,991 \pm 50.67^{\mathrm{a}}$ & $2,972 \pm 43.73^{\mathrm{a}}$ & $2,842 \pm 61.93^{\mathrm{b}}$ & $3,087 \pm 108.10$ & $3,167 \pm 98.34$ & $3,157 \pm 132.62$ \\
\hline Protein (first $100 \mathrm{~d}$ ), \% & $3.07 \pm 0.04^{\mathrm{a}}$ & $3.02 \pm 0.04^{\mathrm{b}}$ & $3.01 \pm 0.04^{\mathrm{b}}$ & $3.08 \pm 0.02$ & $3.06 \pm 0.02$ & $3.06 \pm 0.02$ & $3.11 \pm 0.02^{\mathrm{a}}$ & $3.08 \pm 0.02^{\mathrm{ab}}$ & $3.06 \pm 0.02^{\mathrm{b}}$ \\
\hline Fat (first $100 \mathrm{~d}$ ), $\%$ & $3.90 \pm 0.11^{\mathrm{a}}$ & $3.85 \pm 0.10^{\mathrm{b}}$ & $3.73 \pm 0.1^{\mathrm{b}}$ & $3.93 \pm 0.04^{\mathrm{a}}$ & $3.88 \pm 0.04^{\mathrm{b}}$ & $3.75 \pm 0.05^{\mathrm{a}}$ & $3.74 \pm 0.05^{\mathrm{a}}$ & $3.70 \pm 0.04^{\mathrm{a}}$ & $3.52 \pm 0.06^{\mathrm{b}}$ \\
\hline Milk, $\mathrm{kg}$ & $9,041 \pm 555.84^{\mathrm{a}}$ & $9,117 \pm 549.23^{\mathrm{a}}$ & $8,596 \pm 536.77^{\mathrm{b}}$ & $8,984 \pm 189.19^{\mathrm{a}}$ & $8,901 \pm 170.35^{\mathrm{a}}$ & $8,252 \pm 212.07^{\mathrm{b}}$ & $9,275 \pm 223.82^{\mathrm{a}}$ & $9,289 \pm 220.57^{\mathrm{a}}$ & $8,811 \pm 251.16^{\mathrm{b}}$ \\
\hline Protein, \% & $3.21 \pm 0.07^{\mathrm{a}}$ & $3.13 \pm 0.07^{\mathrm{ab}}$ & $3.13 \pm 0.07^{\mathrm{b}}$ & $3.26 \pm 0.02$ & $3.23 \pm 0.02$ & $3.23 \pm 0.03$ & $3.27 \pm 0.03^{\mathrm{a}}$ & $3.21 \pm 0.03^{\mathrm{b}}$ & $3.20 \pm 0.03^{\mathrm{b}}$ \\
\hline Fat, $\%$ & $3.71 \pm 0.16^{\mathrm{a}}$ & $3.65 \pm 0.16^{\mathrm{ab}}$ & $3.56 \pm 0.16^{\mathrm{b}}$ & $3.79 \pm 0.06^{\mathrm{a}}$ & $3.73 \pm 0.05^{\mathrm{ab}}$ & $3.68 \pm 0.07^{\mathrm{b}}$ & $3.64 \pm 0.07^{\mathrm{a}}$ & $3.63 \pm 0.06^{\mathrm{b}}$ & $3.48 \pm 0.07^{\mathrm{b}}$ \\
\hline Services per conception & $2.15 \pm 0.48$ & $2.15 \pm 0.43$ & $1.98 \pm 0.43$ & $2.60 \pm 0.22^{\mathrm{a}}$ & $2.24 \pm 0.17^{\mathrm{ab}}$ & $1.83 \pm 0.25^{\mathrm{b}}$ & $1.84 \pm 0.29$ & $1.97 \pm 0.26$ & $1.65 \pm 0.36$ \\
\hline Days open, d & $155.92 \pm 19.25$ & $139.88 \pm 17.43$ & $149.82 \pm 17.45$ & $142.88 \pm 9.61^{\mathrm{a}}$ & $126.32 \pm 8.19^{\mathrm{b}}$ & $122.76 \pm 10.97^{\mathrm{b}}$ & $126.75 \pm 9.50$ & $111.84 \pm 7.48$ & $114.93 \pm 10.42$ \\
\hline Calving interval, $\mathrm{d}$ & $433.26 \pm 20.87^{\mathrm{a}}$ & $413.68 \pm 18.79^{\mathrm{b}}$ & $426.30 \pm 19.10^{\mathrm{a}}$ & $421.65 \pm 9.45^{\mathrm{a}}$ & $401.34 \pm 7.73^{\mathrm{b}}$ & $399.82 \pm 10.56^{\mathrm{b}}$ & $403.95 \pm 9.19^{\mathrm{a}}$ & $383.52 \pm 7.02^{\mathrm{b}}$ & $390.75 \pm 9.54^{\mathrm{ab}}$ \\
\hline \multicolumn{10}{|l|}{ Second lactation } \\
\hline Milk, kg & $10,050 \pm 589.48^{\mathrm{a}}$ & $9,878 \pm 550.76^{\mathrm{ab}}$ & $9,421 \pm 547.38^{\mathrm{b}}$ & $10,401 \pm 237.96$ & $10,095 \pm 204.53$ & $9,368 \pm 273.51$ & $10,880 \pm 252.81^{\mathrm{a}}$ & $10,744 \pm 199.09^{\mathrm{a}}$ & $10,223 \pm 282.44^{\mathrm{b}}$ \\
\hline Protein, $\%$ & $3.22 \pm 0.06$ & $3.20 \pm 0.06$ & $3.25 \pm 0.06$ & $3.23 \pm 0.03$ & $3.24 \pm 0.02$ & $3.28 \pm 0.03$ & $3.25 \pm 0.03$ & $3.24 \pm 0.02$ & $3.26 \pm 0.03$ \\
\hline Fat, $\%$ & $3.70 \pm 0.14$ & $3.68 \pm 0.13$ & $3.67 \pm 0.13$ & $3.62 \pm 0.06$ & $3.70 \pm 0.05$ & $3.64 \pm 0.07$ & $3.58 \pm 0.06$ & $3.61 \pm 0.05$ & $3.52 \pm 0.07$ \\
\hline Days open, $\mathrm{d}$ & $159.59 \pm 28.95$ & $146.06 \pm 27.18$ & $138.12 \pm 26.91$ & $154.44 \pm 12.12^{\mathrm{a}}$ & $157.59 \pm 10.56^{\mathrm{a}}$ & $132.45 \pm 14.11^{\mathrm{b}}$ & $121.77 \pm 13.27$ & $132.92 \pm 12.07$ & $115.21 \pm 16.28$ \\
\hline $\begin{array}{l}\text { Calving interval, d } \\
\text { Third lactation }\end{array}$ & Third lactation & $396.89 \pm 8.89$ & $386.81 \pm 12.19$ & $420.62 \pm 14.93$ & $413.39 \pm 14.28$ & $393.93 \pm 17.33$ & $391.86 \pm 15.09$ & $395.25 \pm 14.31$ & $381.77 \pm 18.47$ \\
\hline Milk, kg & $11,351 \pm 929.61^{\mathrm{a}}$ & $10,845 \pm 885.53^{\mathrm{a}}$ & $10,008 \pm 847.61^{\mathrm{b}}$ & $11,029 \pm 340.52^{\mathrm{a}}$ & $10,964 \pm 312.30^{\mathrm{a}}$ & $9,915 \pm 416.21^{\mathrm{b}}$ & $11,384 \pm 354.13^{\mathrm{a}}$ & $11,082 \pm 277.65^{\mathrm{a}}$ & $10,095 \pm 385.26^{\mathrm{b}}$ \\
\hline Protein, \% & $3.12 \pm 0.10$ & $3.11 \pm 0.10$ & $3.13 \pm 0.09$ & $3.20 \pm 0.04$ & $3.19 \pm 0.03$ & $3.21 \pm 0.05$ & $3.23 \pm 0.04$ & $3.23 \pm 0.03$ & $3.22 \pm 0.04$ \\
\hline Fat, $\%$ & $3.53 \pm 0.25$ & $3.52 \pm 0.24$ & $3.55 \pm 0.23$ & $3.64 \pm 0.10$ & $3.67 \pm 0.09$ & $3.61 \pm 0.12$ & $3.56 \pm 0.10$ & $3.54 \pm 0.08$ & $3.57 \pm 0.11$ \\
\hline \multicolumn{10}{|l|}{$\mathrm{LP}^{2}$} \\
\hline Milk, kg & $10,243 \pm 718^{\mathrm{a}}$ & $9,915 \pm 684^{\mathrm{a}}$ & $9,315 \pm 655^{\mathrm{b}}$ & $9,957 \pm 258^{\mathrm{a}}$ & $9,992 \pm 236^{\mathrm{a}}$ & $9,012 \pm 315^{\mathrm{b}}$ & $10,366 \pm 276^{\mathrm{a}}$ & $10,320 \pm 216^{\mathrm{a}}$ & $9,529 \pm 300^{\mathrm{b}}$ \\
\hline Protein, $\%$ & $3.16 \pm 0.09$ & $3.12 \pm 0.08$ & $3.11 \pm 0.08$ & $3.21 \pm 0.03$ & $3.18 \pm 0.03$ & $3.21 \pm 0.04$ & $3.25 \pm 0.03$ & $3.19 \pm 0.03$ & $3.19 \pm 0.04$ \\
\hline Fat, $\%$ & $3.73 \pm 0.22$ & $3.65 \pm 0.21$ & $3.65 \pm 0.20$ & $3.75 \pm 0.08$ & $3.73 \pm 0.08$ & $3.63 \pm 0.10$ & $3.63 \pm 0.08$ & $3.52 \pm 0.06$ & $3.50 \pm 0.09$ \\
\hline
\end{tabular}

Oु. $\quad$ ac Within a row, mean values related to the same explanatory variable with different superscript letters are significantly different $(P<0.05)$.

紊 $\quad{ }^{1}$ Age at first calving.

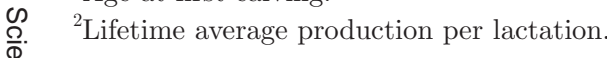


Table 5. Effects of average age at first calving (AFC), BCS (5-point scale), and BW on production and reproduction outcomes

\begin{tabular}{|c|c|c|c|c|c|c|c|c|c|}
\hline \multirow[b]{2}{*}{ Item } & \multicolumn{3}{|c|}{$\mathrm{AFC}, \mathrm{d}$} & \multicolumn{3}{|c|}{ BCS } & \multicolumn{3}{|c|}{$\mathrm{BW}, \mathrm{kg}$} \\
\hline & $\geq 751$ & $750-700$ & $\leq 699$ & 14 mo of age & $\begin{array}{c}14 \text { mo of age } \\
(3.5-3.25)\end{array}$ & $14 \underset{(\leq 3)}{1}$ mo of age & 14 mo of age & $\begin{array}{c}14 \text { mo of age } \\
(419-380)\end{array}$ & $14 \underset{(\leq 379)}{\text { mo of age }}$ \\
\hline $\mathrm{n}$ & 215 & 296 & 269 & 90 & 268 & 172 & 343 & 302 & 135 \\
\hline BCS at 14 mo of age, 5 -point scale & - & - & - & - & - & - & $3.48 \pm 0.02^{\mathrm{a}}$ & $3.33 \pm 0.02^{\mathrm{b}}$ & $3.19 \pm 0.03^{\mathrm{c}}$ \\
\hline $\mathrm{BW}$ at $14 \mathrm{mo}$ of age, $\mathrm{kg}$ & - & - & - & $431.92 \pm 3.84^{\mathrm{a}}$ & $417.42 \pm 2.05^{\mathrm{b}}$ & $394.77 \pm 2.78^{c}$ & - & - & - \\
\hline Services per conception (heifers) & - & - & - & $1.81 \pm 0.20$ & $1.87 \pm 0.12$ & $2.02 \pm 0.15$ & $1.94 \pm 0.11$ & $1.91 \pm 0.11$ & $1.81 \pm 0.16$ \\
\hline $\mathrm{AFC}, \mathrm{d}$ & & & & $724.78 \pm 7.14$ & $727.58 \pm 3.81$ & $735.52 \pm 5.17$ & $721.07 \pm 3.39^{c}$ & $736.26 \pm 3.42^{\mathrm{b}}$ & $757.95 \pm 5.25^{\mathrm{a}}$ \\
\hline \multicolumn{10}{|l|}{ First lactation } \\
\hline Milk (first $100 \mathrm{~d}$ ), $\mathrm{kg}$ & $3,046 \pm 32.29^{\mathrm{a}}$ & $2,961 \pm 27.36^{\mathrm{b}}$ & $2,917 \pm 32.04^{\mathrm{b}}$ & $2,886 \pm 63.93$ & $2,965 \pm 34.52$ & $2,972 \pm 46.11$ & $3,001 \pm 32.65^{\mathrm{a}}$ & $2,967 \pm 34.35^{\mathrm{ab}}$ & $2,881 \pm 51.78^{\mathrm{b}}$ \\
\hline Protein (first $100 \mathrm{~d}$ ), \% & $3.07 \pm 0.01^{\mathrm{b}}$ & $3.09 \pm 0.01^{\mathrm{a}}$ & $3.11 \pm 0.01^{\mathrm{a}}$ & $3.12 \pm 0.02$ & $3.10 \pm 0.01$ & $3.08 \pm 0.02$ & $3.09 \pm 0.01$ & $3.08 \pm 0.01$ & $3.08 \pm 0.2$ \\
\hline Fat (first $100 \mathrm{~d}$ ), \% & $3.93 \pm 0.03^{\mathrm{a}}$ & $3.82 \pm 0.03^{\mathrm{b}}$ & $3.83 \pm 0.03^{\mathrm{b}}$ & $3.98 \pm 0.06^{\mathrm{a}}$ & $3.84 \pm 0.03^{\mathrm{b}}$ & $3.74 \pm 0.04^{\mathrm{c}}$ & $3.92 \pm 0.03^{\mathrm{a}}$ & $3.85 \pm 0.03^{\mathrm{a}}$ & $3.71 \pm 0.05^{\mathrm{b}}$ \\
\hline Milk, kg & $8,946 \pm 133.25$ & $8,816 \pm 129.85$ & $8,800 \pm 136.95$ & $8,516 \pm 234.74$ & $8,848 \pm 166.58$ & $8,806 \pm 194.68$ & $8,977 \pm 139.59^{\mathrm{a}}$ & $8,822 \pm 141.04^{\mathrm{a}}$ & $8,346 \pm 180.70^{\mathrm{b}}$ \\
\hline Protein, \% & $3.24 \pm 0.02$ & $3.24 \pm 0.02$ & $3.26 \pm 0.02$ & $3.32 \pm 0.03^{\mathrm{a}}$ & $3.25 \pm 0.02^{\mathrm{b}}$ & $3.23 \pm 0.03^{\mathrm{b}}$ & $3.26 \pm 0.02$ & $3.23 \pm 0.02$ & $3.22 \pm 0.02$ \\
\hline Fat, $\%$ & $3.81 \pm 0.04$ & $3.75 \pm 0.04$ & $3.79 \pm 0.04$ & $3.89 \pm 0.07^{\mathrm{a}}$ & $3.79 \pm 0.05^{\mathrm{b}}$ & $3.70 \pm 0.06^{\mathrm{a}}$ & $3.80 \pm 0.04^{\mathrm{a}}$ & $3.79 \pm 0.04^{\mathrm{a}}$ & $3.70 \pm 0.06^{\mathrm{b}}$ \\
\hline Services per conception & $2.25 \pm 0.15$ & $2.24 \pm 0.11$ & $2.21 \pm 0.14$ & $1.86 \pm 0.26$ & $2.19 \pm 0.16$ & $2.33 \pm 0.23$ & $2.35 \pm 0.14$ & $2.23 \pm 0.14$ & $1.91 \pm 0.23$ \\
\hline Days open, d & $145.78 \pm 7.24^{\mathrm{a}}$ & $139.35 \pm 6.29^{\mathrm{b}}$ & $132.38 \pm 6.96^{\mathrm{b}}$ & $105.87 \pm 13.2^{\mathrm{b}}$ & $138.84 \pm 7.62^{\mathrm{a}}$ & $153.81 \pm 10.0^{\mathrm{a}}$ & $140.80 \pm 6.84$ & $132.51 \pm 7.11$ & $136.43 \pm 10.28$ \\
\hline Calving interval, $\mathrm{d}$ & $418.82 \pm 7.78$ & $408.48 \pm 6.10$ & $407.80 \pm 6.99$ & $388.27 \pm 13.10^{\mathrm{b}}$ & $414.11 \pm 7.39^{\mathrm{a}}$ & $428.23 \pm 10.58^{\mathrm{a}}$ & $415.77 \pm 7.04$ & $403.92 \pm 6.91$ & $411.44 \pm 10.01$ \\
\hline \multicolumn{10}{|l|}{ Second lactation } \\
\hline Milk, kg & $10,221 \pm 196.81$ & $10,220 \pm 153.83$ & $10,449 \pm 173.75$ & $9,648 \pm 311.66^{\mathrm{b}}$ & $10,314 \pm 193.37^{\mathrm{a}}$ & $10,086 \pm 260.53^{\mathrm{ab}}$ & $10,519 \pm 177.44^{\mathrm{a}}$ & $10,219 \pm 190.68^{\mathrm{a}}$ & $9,671 \pm 253.10^{\mathrm{b}}$ \\
\hline Protein, \% & $3.28 \pm 0.02$ & $3.24 \pm 0.02$ & $3.24 \pm 0.02$ & $3.34 \pm 0.04^{\mathrm{a}}$ & $3.26 \pm 0.02^{\mathrm{b}}$ & $3.27 \pm 0.03^{\mathrm{ab}}$ & $3.24 \pm 0.02$ & $3.25 \pm 0.02$ & $3.24 \pm 0.03$ \\
\hline Fat, $\%$ & $3.71 \pm 0.05$ & $3.67 \pm 0.04$ & $3.65 \pm 0.04$ & $3.68 \pm 0.08$ & $3.62 \pm 0.05$ & $3.56 \pm 0.07$ & $3.61 \pm 0.04$ & $3.69 \pm 0.05$ & $3.61 \pm 0.06$ \\
\hline Days open, $\mathrm{d}$ & $159.17 \pm 10.21$ & $147.54 \pm 8.63$ & $150.12 \pm 9.07$ & $135.91 \pm 16.62$ & $147.61 \pm 10.73$ & $141.88 \pm 14.73$ & $157.40 \pm 9.46^{\mathrm{a}}$ & $156.51 \pm 9.64^{\mathrm{a}}$ & $126.08 \pm 12.4^{\mathrm{b}}$ \\
\hline Calving interval, $\mathrm{d}$ & $431.87 \pm 10.87^{\mathrm{a}}$ & $409.58 \pm 8.93^{\mathrm{b}}$ & $430.73 \pm 10.29^{\mathrm{a}}$ & $432.94 \pm 16.40$ & $423.17 \pm 10.48$ & $407.50 \pm 15.07$ & $433.43 \pm 9.58^{\mathrm{a}}$ & $417.64 \pm 9.53^{\mathrm{ab}}$ & $394.75 \pm 13.57^{\mathrm{b}}$ \\
\hline \multicolumn{10}{|l|}{ Third lactation } \\
\hline Milk, kg & $9,903 \pm 281.97^{\mathrm{b}}$ & $10,570 \pm 237.23^{\mathrm{a}}$ & $10,922 \pm 266 \cdot 26^{\mathrm{a}}$ & $10,710 \pm 456.97$ & $10,577 \pm 312.19$ & $10,695 \pm 428.53$ & $11,057 \pm 246.53^{\mathrm{a}}$ & $10,443 \pm 262.25^{\mathrm{b}}$ & $9,253 \pm 345.12^{\mathrm{c}}$ \\
\hline Protein, \% & $3.22 \pm 0.02^{\mathrm{a}}$ & $3.15 \pm 0.02^{\mathrm{b}}$ & $3.17 \pm 0.03^{\mathrm{ab}}$ & $3.25 \pm 0.05$ & $3.24 \pm 0.04$ & $3.22 \pm 0.05$ & $3.18 \pm 0.03$ & $3.18 \pm 0.03$ & $3.18 \pm 0.04$ \\
\hline Fat, $\%$ & $3.75 \pm 0.07^{\mathrm{a}}$ & $3.61 \pm 0.06^{\mathrm{b}}$ & $3.61 \pm 0.07^{\mathrm{b}}$ & $3.63 \pm 0.12$ & $3.59 \pm 0.08$ & $3.55 \pm 0.11$ & $3.65 \pm 0.07$ & $3.64 \pm 0.07$ & $3.65 \pm 0.09$ \\
\hline \multicolumn{10}{|l|}{$\mathrm{LP}^{1}$} \\
\hline Milk, kg & $9,602 \pm 213$ & $9,861 \pm 179$ & $9,859 \pm 201$ & $9,454 \pm 347$ & $9,797 \pm 237$ & $9,952 \pm 326$ & $10,109 \pm 181^{\mathrm{a}}$ & $9,758 \pm 193^{\mathrm{a}}$ & $8,760 \pm 254^{\mathrm{b}}$ \\
\hline Protein, \% & $3.23 \pm 0.03^{\mathrm{a}}$ & $3.17 \pm 0.02^{\mathrm{a}}$ & $3.18 \pm 0.02^{\mathrm{b}}$ & $3.29 \pm 0.05$ & $3.21 \pm 0.03$ & $3.20 \pm 0.04$ & $3.20 \pm 0.02$ & $3.19 \pm 0.03$ & $3.18 \pm 0.03$ \\
\hline Fat, $\%$ & $3.79 \pm 0.07^{\mathrm{a}}$ & $3.67 \pm 0.06^{\mathrm{b}}$ & $3.71 \pm 0.06^{\mathrm{ab}}$ & $3.75 \pm 0.11$ & $3.69 \pm 0.08$ & $3.55 \pm 0.10$ & $3.73 \pm 0.06$ & $3.73 \pm 0.06$ & $3.65 \pm 0.08$ \\
\hline
\end{tabular}

${ }^{\mathrm{a} C \mathrm{c}}$ Within a row, mean values related to the same explanatory variable with different superscript letters are significantly different $(P<0.05)$.

${ }^{1} \mathrm{LP}=$ lifetime average production per lactation 


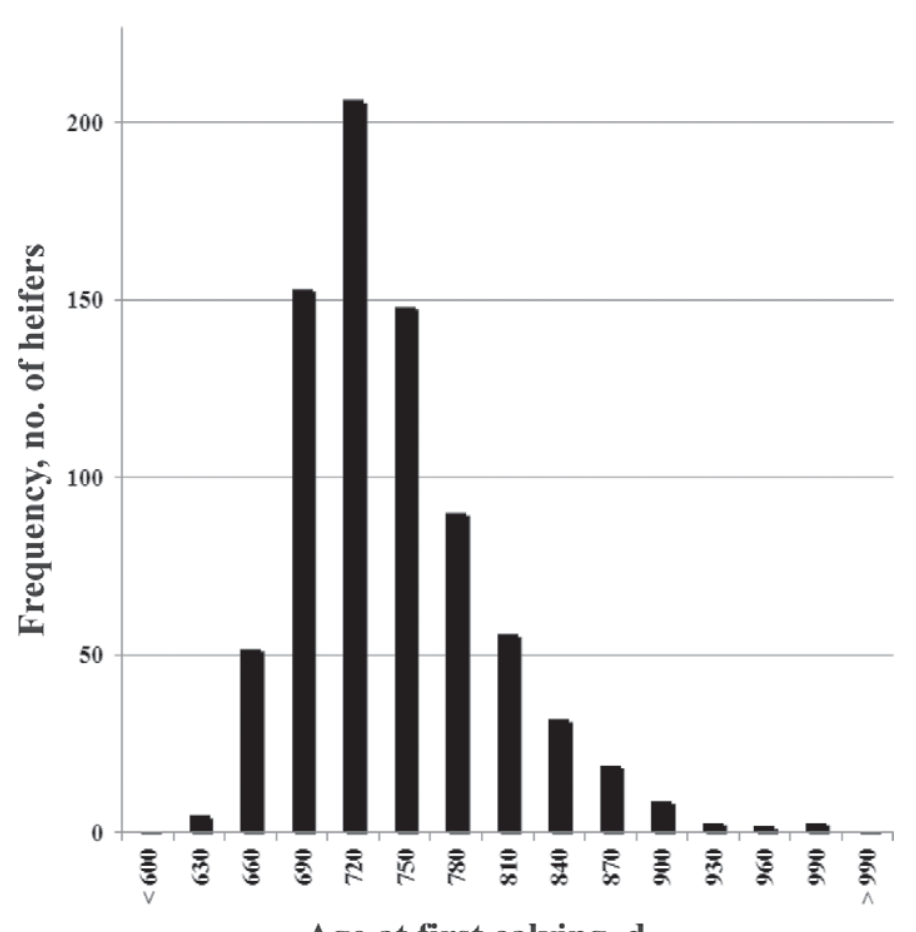

Age at first calving, $d$

Figure 1. Histogram of age at first calving (AFC; d) for all 780 heifers in the study. Ages at first calving were grouped as low $(\leq 699$ $\mathrm{d} ; \mathrm{n}=269$; mean $\pm \mathrm{SD}=674.57 \pm 19.09 \mathrm{~d})$, medium $(750$ to $700 \mathrm{~d} ; \mathrm{n}$ $=296$; mean $\pm \mathrm{SD}=722.88 \pm 14.26 \mathrm{~d})$, and high $(\geq 751 \mathrm{~d} ; \mathrm{n}=215$; mean $\pm \mathrm{SD}=801.35 \pm 48.62 \mathrm{~d}$ ).

observed to have higher ADG. The differences between high ADG $(\geq 0.950 \mathrm{~kg} / \mathrm{d})$ and low ADG $(\leq 0.849 \mathrm{~kg} / \mathrm{d})$ in days open were $20 \mathrm{~d}$ for the first parity and $22 \mathrm{~d}$ for the second parity. Calving interval between the first and second calving reached $414 \mathrm{~d}$ in the medium-ADG (0.969 to $0.850 \mathrm{~kg} / \mathrm{d}$ ) compared with $433 \mathrm{~d}$ in the highADG $(\geq 0.970 \mathrm{~kg} / \mathrm{d})$ group and $426 \mathrm{~d}$ in the low-ADG $(\leq 0.849 \mathrm{~kg} / \mathrm{d} ; P<0.05)$ group. Similar results of ADG effects, but with lower differences, were observed between the other evaluated periods (Table 4 ).

\section{$B C S, B W$, and $A F C$}

The higher the BW at 14 mo of age $(\geq 420 \mathrm{~kg})$, the higher the milk yield in the first $(8,977 \mathrm{~kg})$, second $(10,519 \mathrm{~kg})$, and third $(11,057 \mathrm{~kg})$ parity, and the higher the average LP $(10,109 \mathrm{~kg} ; P<0.05)$. The higher the BW at 14 mo of age, the lower the AFC $(721 \mathrm{~d} ; P$ $<0.05$; Table 5). The evaluated groups of BCS at 14 mo showed no differences between AFC or milk yield in the first lactation and average LP. However, the components in milk (fat and protein) in the first parity differed $(P<0.05)$ according to the level of BCS at 14 mo of age. The higher the BCS $(\geq 3.75)$, the higher the content of milk components detected $(3.32 \%$ protein and $3.89 \%$ fat $)$. A significant difference $(P<0.05)$ in the days open in the first parity $(-48 \mathrm{~d})$ and calving interval between first and second parity $(-40 \mathrm{~d})$ was found between the highest BCS $(\geq 3.75)$ and the lowest $\operatorname{BCS}(\leq 3)$.

The following section presents regression and correlation coefficients between BCS at 14 mo of age and all evaluated periods of ADG (Table 2). The regression and correlation coefficients for the relationships between BCS and prepubertal ADG (5 to 10 mo of age) were a BCS of $1.13(P<0.001)$ and $0.33(P<0.001)$, respectively; between BCS and postpubertal ADG (11 to 14 mo of age) were a BCS of $1.91(P<0.001)$ and $0.45(P<0.001)$, respectively; and between BCS and total ADG (5 to 14 mo of age) were a BCS of 1.64 $(P<0.001)$ and $0.41(P<0.001)$, respectively. These coefficients showed a medium level of correlation, indicating that a medium relationship between the evaluated variables exists. For example, postpubertal growth calculated from 11 to 14 mo of age had the greatest influence on BCS with a level of correlation of 0.45. The regression coefficient was a BCS of 1.91, indicating that a 1-kg increase in ADG would lead to an increase in BCS of 1.91 in the above-mentioned period of growth.

\section{AFC}

The distribution of AFC is shown in Figure 1. The overall mean and standard deviation of AFC was 728 $\pm 57.72 \mathrm{~d}$ (Table 2). The AFC level had a significant effect on milk yield in the first $100 \mathrm{~d}$ of the first lactation $(P<0.05)$. The group of cows with AFC $\geq 751 \mathrm{~d}$ produced $3,046 \mathrm{~kg}$ of milk/100 d, whereas the group with AFC $\leq 699$ d produced only 2,917 kg/100 d (Table $5)$. The highest milk fat content, which was found at the highest AFC ( $\geq 751 \mathrm{~d})$, differed significantly from the other groups $(P<0.05)$. Milk protein was significantly the lowest at the highest AFC. No differences between groups were observed in milk yield and milk components content for the second parity. The groups differed in the third parity, showing the highest milk yield $(10,922 \mathrm{~kg} ; P<0.05)$ for the lowest AFC group accompanied by a lower level of milk protein and fat (3.17 and $3.61 \%$, respectively; $P>0.05$ ). The lowest milk yield in LP occurred with an AFC $\geq 751$ d. Days open in the first parity were $14 \mathrm{~d}$ longer in group with $\mathrm{AFC} \geq 751 \mathrm{~d}$ than in the group with $\mathrm{AFC} \leq 699 \mathrm{~d}$ and $6 \mathrm{~d}$ longer than in the group with AFC of 750 to $700 \mathrm{~d}$ $(P<0.05)$.

Growth from 5 to 18 mo of age according to AFC is shown in Figure 2. The ADG increased until 10 mo of age when it reached its maximum $(\mathrm{AFC} \geq 751 \mathrm{~d}$ : $0.96 \pm 0.08 \mathrm{~kg} / \mathrm{d}$, AFC from 750 to $700 \mathrm{~d}: 0.90 \pm 0.10$ $\mathrm{kg} / \mathrm{d}$, and $A F C \leq 699 \mathrm{~d}: 0.89 \pm 0.12 \mathrm{~kg} / \mathrm{d})$ and then 
followed a gradual decline. Changes in BCS during the postpubertal period of growth according to AFC are described in Figure 3. The BCS ranged between 3.2 and 3.5 from 11 to 16 mo of age. The mean and standard deviation for BCS at 14 mo of age (approximate conception period) was $3.36 \pm 0.35$ for $\mathrm{AFC} \geq 751 \mathrm{~d}$, $3.33 \pm 0.32$ for $\mathrm{AFC} 750$ to $700 \mathrm{~d}$, and $3.42 \pm 0.34$ for $\mathrm{AFC} \leq 699 \mathrm{~d}$. The BCS reached 3.5 for the groups with $\mathrm{AFC} \leq 699 \mathrm{~d}$ and $\geq 751 \mathrm{~d}$ at $17 \mathrm{mo}$ of age. The regression and correlation coefficients for the relationship between AFC and prepubertal ADG (5 to 10 mo of age $)$ were $-59.94 \mathrm{~d}(P<0.01)$ and $-0.14(P<0.01)$, respectively; between AFC and postpubertal ADG (11 to 14 mo of age $)$ were $-180.03 \mathrm{~d}(P<0.001)$ and -0.32 $(P<0.001)$, respectively; and between AFC and total ADG $(5$ to 14 mo of age $)$ were $-99.90 \mathrm{~d}(P<0.001)$ and $-0.20(P<0.001)$, respectively. The postpubertal growth had the greatest effect on AFC.

\section{DISCUSSION}

\section{Intensity of Rearing Dairy Heifers}

Growth is one of the fundamental processes that takes place during the life of an animal. It is influenced by genetic potential, nutrition, and environmental conditions

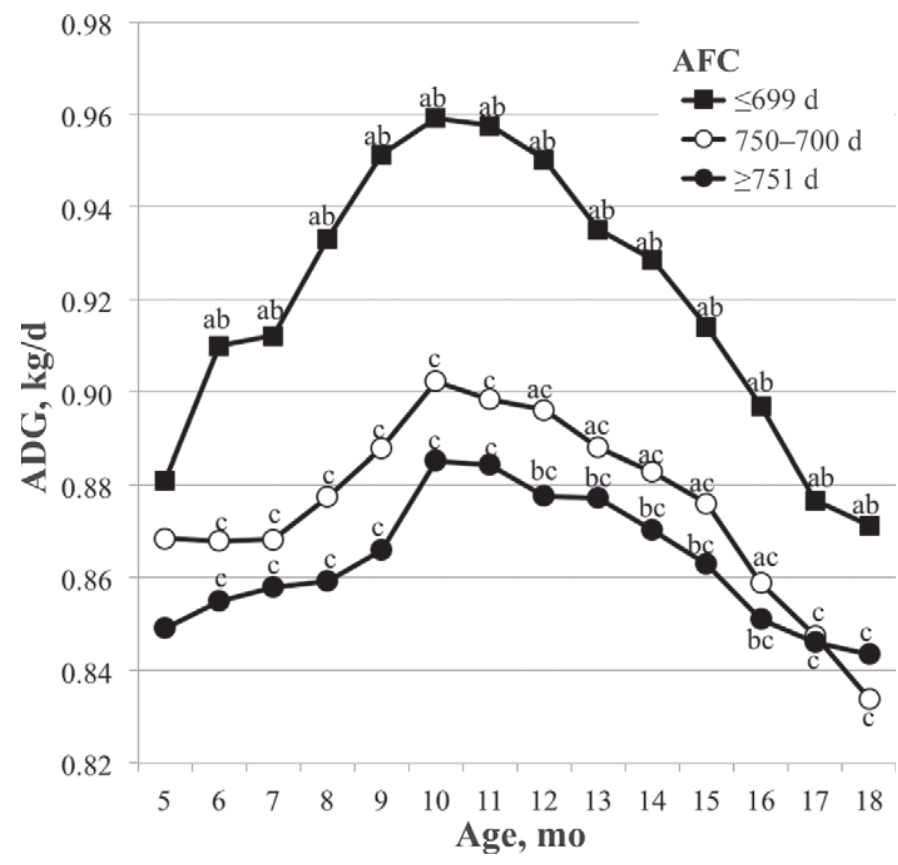

Figure 2. Growth of heifers (ADG in $\mathrm{kg} / \mathrm{d}$ ) according to age at first calving (AFC). Ages at calving were grouped as high ( $\geq 751 \mathrm{~d} ; \mathrm{n}$ $=215$; mean $\pm \mathrm{SD}=801.35 \pm 48.62 \mathrm{~d})$, medium $(750$ to $700 \mathrm{~d} ; \mathrm{n}=$ 296 ; mean $\pm \mathrm{SD}=722.88 \pm 14.26 \mathrm{~d})$, and low $(\leq 699 \mathrm{~d} ; \mathrm{n}=269$; mean $\pm \mathrm{SD}=674.57 \pm 19.09 \mathrm{~d})$. Differences between groups $(P<0.001)$ are denoted with different letters $(\mathrm{a}-\mathrm{c})$ vertically.

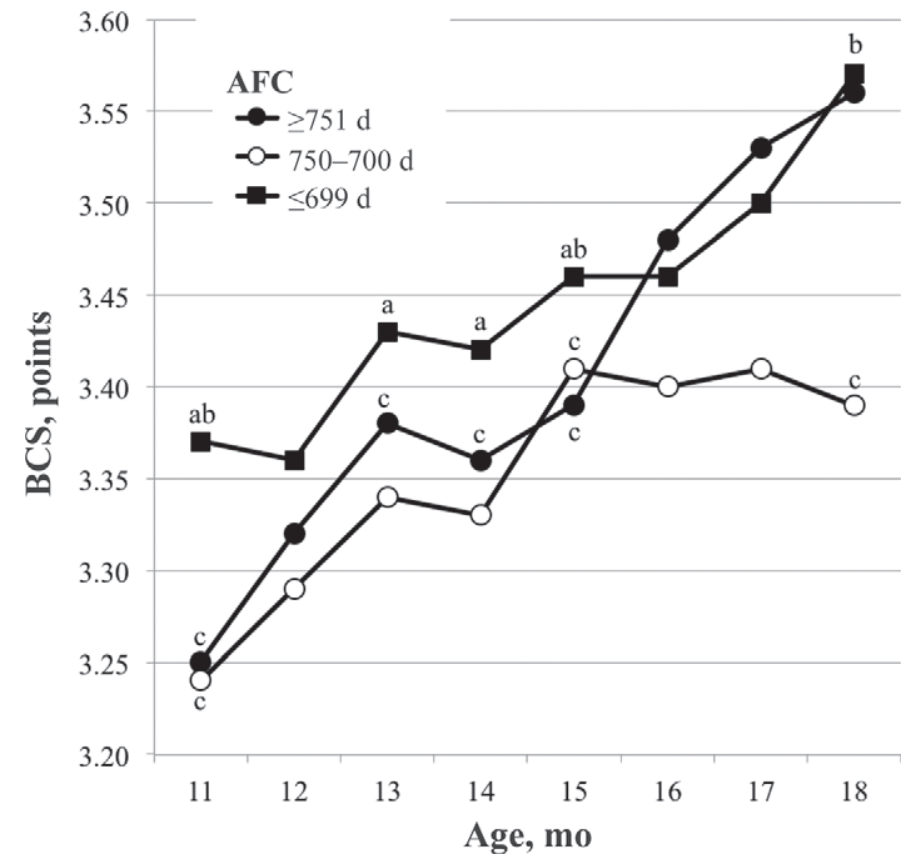

Figure 3. Body condition score in the postpubertal period of growth according to age at first calving (AFC). Ages at calving were grouped as high ( $\geq 751 \mathrm{~d} ; \mathrm{n}=215$; mean $\pm \mathrm{SD}=801.35 \pm 48.62 \mathrm{~d}$ ), medium ( 750 to $700 \mathrm{~d} ; \mathrm{n}=296$; mean $\pm \mathrm{SD}=722.88 \pm 14.26 \mathrm{~d}$ ), and low ( $\leq 699 \mathrm{~d} ; \mathrm{n}=269 ;$ mean $\pm \mathrm{SD}=674.57 \pm 19.09 \mathrm{~d})$. Differences between groups $(P<0.05)$ are denoted with different letters $(\mathrm{a}-\mathrm{c})$ vertically.

(Mourits et al., 2000). The onset of a heifer's sexual maturity depends more on BW (Froidmont et al., 2013) and fat percentage (Abeni et al., 2000) than age and can be significantly influenced by the level of nutrition. According to Silva et al. (2002) the body's cells and parenchyma cells of the mammary gland develop at similar rates in the period from birth to 3 mo of age, but the parenchyma of the mammary gland develops 3 to 4 times faster than the cells of the body in the period from 3 to 9 mo of age (prepubertal period). Most studies have documented the negative effect of high ADG during the heifer rearing period on milk production in the first lactation and especially in the prepubertal period, due to the reduction of parenchyma growth in the mammary gland (Sejrsen and Purup, 1997; Silva et al., 2002; Ettema and Santos, 2004; Daniels, 2010), although some experiments did not confirm this relationship (Kertz et al., 1984; Waldo et al., 1998; Pirlo et al., 2000), or only in cases where the ADG exceeded $0.7 \mathrm{~kg} / \mathrm{d}$ (Abeni et al., 2000) or $0.9 \mathrm{~kg} / \mathrm{d}$ (Mourits et al., 1999b; Whitlock et al., 2002). Moallem et al. (2010) gave an upper limit threshold of $0.8 \mathrm{~kg} / \mathrm{d}$ for the optimal development of the mammary gland and maximal production, and it was validated by a meta-analysis of 8 studies (Zanton and Heinrichs, 2005). Van Amburgh 
et al. (1998) reported a decrease in milk production during subsequent lactations from 10 to $40 \%$ in relation to low $(\leq 0.4 \mathrm{~kg} / \mathrm{d})$ or high $(>0.8 \mathrm{~kg} / \mathrm{d})$ ADG before puberty. Shamay et al. (2005) concluded that an ADG of about $0.7 \mathrm{~kg} / \mathrm{d}$ is optimal for achieving maximum performance. In the current study, $\mathrm{ADG} \geq 0.970 \mathrm{~kg} / \mathrm{d}$ in the prepubertal period ( 5 to 10 mo of age) and ADG $\geq 0.950 \mathrm{~kg} / \mathrm{d}$ in the total period ( 5 to 14 mo of age) showed a negative influence on subsequent milk production in the first lactation. The lowest milk yield $(P$ $<0.05)$ in all the evaluated groups was detected in the first lactation with $\mathrm{ADG} \leq 0.849 \mathrm{~kg} / \mathrm{d}$. According to Tozer and Heinrichs (2001) and Sakaguchi et al. (2005), the negative effect of high levels of nutrition before puberty occurs in all breeds. According to Madgwick et al. (2005), accelerated growth of Holstein heifers during puberty, supported by a higher concentration of protein in the diet, does not reduce subsequent milk production. The same conclusion is supported by Macdonald et al. (2005), who stated that heifers with faster growth during the prepubertal period had reduced development of the mammary glands, but milk yield did not decline because of better physical readiness for the first lactation caused by previous faster growth. Le Cozler et al. (2008) added that heifers with a high potential for milk production appear to be less sensitive to significant negative effects of the high level of nutrition and also that faster growth has a positive effect on the attainment of puberty. In this study, faster growth led to earlier AFC, which was related to earlier maturity of these animals. Foldager and Sejrsen (1991) stated that an increase in ADG from 0.4 to $0.6 \mathrm{~kg} / \mathrm{d}$ during the heifer rearing led to increased subsequent milk production and mammary gland size by $10 \%$. Further increase in the ADG to $0.8 \mathrm{~kg} / \mathrm{d}$ had no effect on the mammary gland volume or milk production. According to Abeni et al. (2000), an average weight gain of 0.9 $\mathrm{kg} / \mathrm{d}$ significantly reduces the milk fat content. The results presented in Table 4 demonstrate that, in the current study, high ADG did not reduce fat or protein content in milk. Heifers with the highest ADG $(\geq 0.950$ $\mathrm{kg} / \mathrm{d}$ ) in the rearing period (prepubertal growth $\geq 0.970$ $\mathrm{kg} / \mathrm{d}$ ) also had the highest components in milk in the first lactation. Hohenboken et al. (1995) argued that the relationship between ADG and milk production depends on the breed and its genetic potential. Smaller breeds are more sensitive to the undesirable effects of intensive rearing in relation to performance in the first lactation (Foldager and Sejrsen, 1991). Kratochvilova (2001) added that growth intensity is highly variable, lowly heritable, and a poor indicator of subsequent milk production. Low correlation between ADG and milk yield found in the current study corroborate previous findings.

\section{Optimal AFC}

Views on the optimal AFC are not completely uniform (Mourits et al., 2000). The extension of the rearing period generally caused a negative increase in age (and weight) at first conception and calving (Stevenson et al., 2008). In the current study, as expected, the lowest AFC was achieved with the highest ADG (Table 4). The difference between high ADG $(\geq 0.950 \mathrm{~kg} / \mathrm{d})$ and low ADG $(\leq 0.849 \mathrm{~kg} / \mathrm{d})$ in length of the rearing period was 22 d. According to Heinrichs and Gabler (2003), the AFC is influenced by events around the birth as well as nutrition, health, and environmental factors operating during the first 4 mo of life.

Mourits et al. (2000) found that increasing the ADG above $0.9 \mathrm{~kg} / \mathrm{d}$ in prepubertal heifers and a maximum achievement of ADG of $1.1 \mathrm{~kg} / \mathrm{d}$ during the postpubertal period enables reaching the first calving at 20.5 mo of age with $563 \mathrm{~kg}$ of $\mathrm{BW}$, which equaled feed savings of $\$ 107 /$ heifer per year. Our results indicate that an approximate AFC of 23.5 mo of age could be achieved by a total ADG higher than $0.950 \mathrm{~kg} / \mathrm{d}(5 \mathrm{mo}$ to 14 mo of age; Table 4). Mourits et al. (1999a) found no disadvantages of early calving before 22 mo of age, when the growth was maintained and controlled in the heifer rearing period. Ettema and Santos (2004) added that early-calved heifers have lower yields in the first lactation, but higher lifetime performance. Results in Table 4 point to a lower milk yield in the first lactation due to higher ADG (prepubertal growth $\geq 0.970$ $\mathrm{kg} / \mathrm{d}$ and total growth $\geq 0.950 \mathrm{~kg} / \mathrm{d}$ ) compared with medium growth, but animals with higher ADG in the rearing period had a higher milk yield in the second and third lactations than animals with medium or low ADG. The highest LP occurred with groups of prepubertal growth $\geq 0.970 \mathrm{~kg} / \mathrm{d}$ and total growth $\geq 0.950$ $\mathrm{kg} / \mathrm{d}$. This finding is consistent with the fact that high ADG does not negatively affect the LP. Similar results were found by Dawson and Carson (2004), who stated that heifers that calved at $540 \mathrm{~kg}$ were more economical than those at $620 \mathrm{~kg}$. Late-calved heifers produced $11 \%$ more milk in the first lactation, and early-calved heifers lost more weight in early lactation and their calving interval was extended. Nonetheless, this fact had no effect on milk production in the second and third lactation. Froidmont et al. (2013) confirmed the strong positive relationship between BW at first calving and milk yield in the first lactation. According to Shamay et al. (2005), Holstein heifers should weigh, on average, $620 \mathrm{~kg}$ during the first month after calving for maximum performance in the first lactation. In the current study, the group with the highest BW $(\geq 420$ $\mathrm{kg}$ ) at the first insemination achieved the highest milk yield in the first, second, and third parities (Table 5). 
According to Hoffman et al. (1996), intensive growth with low efficiency of insemination may lead to negative consequences. Intensive rearing of heifers can lead to calving at 22 mo of age. However, heifers will have undesirable higher BW and BCS at first calving if they are not pregnant until 15 to 16 mo. These heifers will be extremely susceptible to ketosis, displacement of the abomasum, and low feed intake in the transition period. In the current study, a high ADG $(\geq 0.950$ $\mathrm{kg} / \mathrm{d}$ ) in postpubertal growth negatively influenced the reproduction parameters (Table 4), and high ADG in this period of growth was associated with higher BCS. The BCS from 11 to 16 mo of age (postpubertal period) ranged between 3.2 and 3.5. The BCS at 17 mo of age reached above 3.5 in the group with AFC $\geq 750 \mathrm{~d}$. One of the reasons for increased BCS in the group with $\mathrm{AFC} \geq 750 \mathrm{~d}$ was because heifers were pregnant (Figure 3). The increased fat reserves in the bodies of the heifers could be one of the reasons for the extended AFC. It might have prolonged the age at first conception and subsequent $\mathrm{AFC}$ in the group with $\mathrm{AFC} \geq 751 \mathrm{~d}$. The results also show that the heifers with low BCS $(\leq 3)$ in the conception period (14 mo of age) had reduced reproduction ability in the first parity. However, their calving interval in the second parity was the shortest (407 d) compared with the other groups (Table 5).

Shamay et al. (2005), Ettema and Santos (2004), Heinrichs and Gabler (2003), and Mourits et al. (1999b) suggested that an AFC in the Holstein breed of 23 to $24 \mathrm{mo}$ is optimal for maximum profitable production. Ettema and Santos (2004) found in analyses of age and $\mathrm{BW}$ at first calving for Holsteins that only $2.7 \%$ of dairy farms achieve the recommended targets and, therefore, this leads to economic losses. Wathes et al. (2008) reported that optimal fertility and maintenance of maximum performance in the first lactation were reached at the calving age of 24 to $25 \mathrm{mo}$, although heifers that calved at the age of 22 to 23 mo were the best in overall performance and longevity over $5 \mathrm{yr}$, partly because heifers with good fertility also had a high level of fertility as cows. Abeni et al. (2000) and Van Amburgh et al. (1998) concluded that calving earlier than 23 mo of age is associated with lower milk yields and lower milk fat content, although, it also leads to a higher milk protein content, which is influenced by the reduction in milk production. Moreover, they also concluded that earlier calving also leads to reduced reproduction performance. In our study (Table 5), we did not observe a significant effect of AFC on milk yields, except on milk yield in the first $100 \mathrm{~d}$ of first lactation $(P<0.05)$. We found the highest milk yield in the second lactation, third lactation, and LP for the group with $\mathrm{AFC} \leq 699$.

\section{CONCLUSIONS}

Controlling the AFC is a key factor for ensuring good lifetime milk production and the capability of animals to efficiently produce more milk in less time. The lowest average milk yield in LP was observed in AFC higher than 24.5 mo of age. The highest ADG in all evaluated groups led to a lower AFC of less than 23.5 mo of age. The highest ADG in prepubertal and total growth only influenced milk yield negatively during early first parity, but did not affect negatively the LP. Indeed, highest ADG in prepubertal and total growth were related to higher LP levels. High total growth ADG $(\geq 0.850 \mathrm{~kg} / \mathrm{d})$ was associated with $22 \mathrm{~d}$ lower AFC (approximately 1 estrous cycle of heifers) compared with low ADG $(<0.849 \mathrm{~kg} / \mathrm{d})$. Negative effects of AFC $<23$ mo of age on milk yield traits were not observed, except that milk yield during the first $100 \mathrm{~d}$ of first parity was lower. Adult reproduction performance and milk yields in the second and third parity were the lowest in the group with AFC $>24.5$ mo of age. Prolonged AFC was associated with higher BCS and subsequent potential health problems during the calving period. Results of this study suggest that universal AFC recommendations may not apply to all dairy herds and depend on the animals' performance and level of management at dairy farms. However, the objective of a rearing period leading to an AFC of less than 23 mo of age proves to be a more suitable option for successful rearing of heifers with optimal subsequent production and reproduction in a herd with suitable management.

\section{ACKNOWLEDGMENTS}

We are grateful to Lois Russel for her English manuscript edits. This research was supported by an "S grant of MSMT CR" and by projects 0002701404 from the Ministry of Agriculture (Prague, Czech Republic) and QI91A238 from the National Agency for Agriculture Research (Prague, Czech Republic).

\section{REFERENCES}

Abeni, F., L. Calamari, L. Stefanini, and G. Pirlo. 2000. Effects of daily gain in pre- and postpubertal replacement dairy heifers on BCS, body size, metabolic profile, and future milk production. J. Dairy Sci. 83:1468-1478.

Daniels, K. M. 2010. Dairy heifer mammary development. Pages 69-76 in Proc. 19th Annu. Tri-State Dairy Nutrition Conference. Grand Wayne Center, Fort Wayne, IN.

Dawson, L. E. R., and A. F. Carson. 2004. Management of the dairy heifer. Cattle Pract. 12:181-192.

Edmonson, A. J., I. J. Lean, L. D. Weaver, T. Farver, and G. Webster. 1989. A body condition scoring chart for Holstein dairy cows. J. Dairy Sci. 72:68-78.

Ettema, J. F., and J. E. Santos. 2004. Impact of age at calving on lactation, reproduction, health, and income in first-parity Holsteins on commercial farms. J. Dairy Sci. 87:2730-2742. 
Foldager, J., and K. Sejrsen. 1991. Rearing intensity in dairy heifers and the effect on subsequent milk production. Report No. 693. Institute of Animal Science, Foulum, Denmark.

Ford, J. A., and C. S. Park. 2001. Nutritionally directed compensatory growth enhances heifer development and lactation potential. J. Dairy Sci. 84:1669-1678.

Froidmont, E., P. Mayeres, P. Picron, A. Turlot, V. Planchon, and D. Stilmant. 2013. Association between age at first calving, year and season of first calving and milk production in Holstein cows. Animal 7:665-672.

Gabler, M. T., and A. J. Heinrichs. 2003. Dietary protein to metabolizable energy ratios on feed efficiency and structural growth of prepubertal Holstein heifers. J. Dairy Sci. 86:268-274.

Hoffman, P. C., N. M. Brehm, S. G. Price, and A. Prill-Adams. 1996. Effect of accelerated postpubertal growth and early calving on lactation performance of primiparous Holstein heifers. J. Dairy Sci. 79:2024-2031.

Hohenboken, D., J. Foldager, J. Jensen, P. Madsen, and B. B. Andersen. 1995. Breed and nutritional effects and interactions on energy intake, production and efficiency of nutrient utilization in young bulls, heifers and lactating cows. Acta Agric. Scand. A Anim. Sci. 45:92-98.

ICAR (International Committee for Animal Recording). 2012. International agreement of recording practices; guidelines approved by the General Assembly held in Cork, Ireland on June 2012. Accessed Feb. 27, 2014. http://www.icar.org/Documents/Rules and regulations/Guidelines/Guidelines_2012.pdf.

Kadokawa, H., and G. B. Martin. 2006. A new perspective on management of reproduction in dairy cows: The need for detailed metabolic information, an improved selection index and extended lactation. J. Reprod. Dev. 52:161-168.

Kertz, A. F., L. F. Reutzel, and J. H. Mahoney. 1984. Ad libitum water intake by neonatal calves and its relationship to calf starter intake, weight gain, feces score, and season. J. Dairy Sci. 67:2964-2969.

Kratochvilova, M. 2001. Relationship between growth and milk production in dairy cattle. Czech J. Anim. Sci. 46:139-144.

Le Cozler, Y., V. Lollivier, P. Lacasse, and C. Disenhaus. 2008. Rearing strategy and optimizing first-calving targets in dairy heifers: A review. Animal 2:1393-1404.

Lucy, M. C. 2001. Reproductive loss in high-producing dairy cattle: Where will it end? J. Dairy Sci. 84:1277-1293.

Macdonald, K. A., J. W. Penno, A. M. Bryant, and J. R. Roche. 2005. Effect of feeding level pre- and post-puberty and body weight at first calving on growth, milk production, and fertility in grazing dairy cows. J. Dairy Sci. 88:3363-3375.

Madgwick, S., A. C. Evans, and A. P. Beard. 2005. Treating heifers with GnRH from 4 to 8 weeks of age advanced growth and the age at puberty. Theriogenology 63:2323-2333.

Moallem, U., D. Werner, H. Lehrer, M. Zachut, L. Livshitz, S. Yakoby, and A. Shamay. 2010. Long-term effects of ad libitum whole milk prior to weaning and prepubertal protein supplementation on skeletal growth rate and first-lactation milk production. J. Dairy Sci. 93:2639-2650.

Mourits, M. C. M., D. T. Galligan, A. A. Dijkhuizen, and R. B. M Huirne. 2000. Optimization of dairy heifer management decisions based on production conditions of Pennsylvania. J. Dairy Sci 83:1989-1997.

Mourits, M. C. M., R. B. M. Huirne, A. A. Dijkhuizen, and D. T. Galligan. 1999a. Optimal heifer management decisions and the influence of price and production variables. Livest. Prod. Sci. 60:45-58.

Mourits, M. C. M., R. B. M. Huirne, A. A. Dijkhuizen, A. R. Kristensen, and D. T. Galligan. 1999b. Economic optimization of dairy heifer management decisions. Agric. Syst. 61:17-31.

Pirlo, G., F. Miglior, and M. Speroni. 2000. Effect of age at first calving on production traits and on difference between milk yield returns and rearing costs in Italian Holsteins. J. Dairy Sci. 83:603-608.

Sakaguchi, M., T. Suzuki, Y. Sasamoto, Y. Takahashi, A. Nishiura, and M. Aoki. 2005. Effects of first breeding age on the production and reproduction of Holstein heifers up to the third lactation. Anim. Sci. J. 76:419-426.

SAS Institute. 2008. SAS/STAT ${ }^{\circledR} 9.2$ User's Guide. SAS Institute Inc., Cary, NC.

Sejrsen, K., and S. Purup. 1997. Influence of prepubertal feeding level on milk yield potential of dairy heifers: A review. J. Anim. Sci. $75: 828-835$.

Shamay, A., D. Werner, U. Moallem, H. Barash, and I. Bruckental. 2005. Effect of nursing management and skeletal size at weaning on puberty, skeletal growth rate, and milk production during first lactation of dairy heifers. J. Dairy Sci. 88:1460-1469.

Silva, L. F. P., M. J. VandeHaar, B. K. Whitlock, R. P. Radcliff, and H. A. Tucker. 2002. Short communication: Relationship between body growth and mammary development in dairy heifers. J. Dairy Sci. 85:2600-2602.

Stevenson, J. L., J. A. Rodrigues, F. A. Braga, S. Bitente, J. C. Dalton, J. E. P. Santos, and R. C. Chebel. 2008. Effect of breeding protocols and reproductive tract score on reproductive performance of dairy heifers and economic outcome of breeding programs. J. Dairy Sci. 91:3424-3438.

Tozer, P. R., and A. J. Heinrichs. 2001. What affects the costs of raising replacement dairy heifers: A multiple-component analysis. J. Dairy Sci. 84:1836-1844.

Van Amburgh, M. E., D. M. Galton, D. E. Bauman, R. W. Everett, D. G. L. Fox, E. Chase, and H. N. Erb. 1998. Effects of three prepubertal body growth rates on performance of Holstein heifers during first lactation. J. Dairy Sci. 81:527-538.

Verbeke, G., and G. Molenberghs. 2000. Linear Mixed Models for Longitudinal Data. Springer-Verlag, New York, NY.

Waldo, D. R., A. V. Capuco, and C. E. Rexroad Jr. 1998. Milk production of Holstein heifers fed either alfalfa or corn silage diets at two rates of daily gain. J. Dairy Sci. 81:756-764.

Wathes, D. C., J. S. Brickell, N. E. Bourne, A. Swali, and Z. Cheng. 2008. Factors influencing heifer survival and fertility on commercial dairy farms. Animal 2:1135-1143.

Whitlock, B. K., M. J. VandeHaar, L. F. P. Silva, and H. A. Tucker. 2002. Effect of dietary protein on prepubertal mammary development in rapidly growing dairy heifers. J. Dairy Sci. 85:1516-1525.

Zanton, G. I., and A. J. Heinrichs. 2005. Meta-analysis to assess effect of prepubertal average daily gain of Holstein heifers on firstlactation production. J. Dairy Sci. 88:3860-3867. 